\title{
Bioanalysis
}

\section{Activity-based proteomics in bioanalysis: past, present and future}

\section{"The recent advancements in the technology of LC-MS will definitely take activity-based proteomics up to a new height in the field of bioanalysis."}

\begin{abstract}
First draft submitted: 7 March 2017; Accepted for publication: 13 March 2017;
\end{abstract} Published online: 18 May 2017

Keywords: • activity-based probe $\bullet$ bioanalytical technologies $\bullet$ mass spectrometry - proteomics

Traditionally, a high-throughput characterization of proteins in a biological system using state-of-art technologies such as robotics and MS along with other bioinformatics tools is termed as 'proteomics'. Bioanalytical scientists have seen the emergence of diverse range of strategies to solve specific targeted problems as the research in proteomics has matured. Since, the inception of proteomics it has faced a serious setback that it could only determine the amount, but not the activity of a given enzyme present in a cell and hence it has prohibited any direct conclusion with respect to protein function.

About a decade ago [1,2], activity-based proteomics (abP) emerged as one such unique way which could overcome this limitation of proteomics by providing a fast, sensitive and selective solution for identification and enrichment (biotin) of less abundant 'hidden' proteins from the complex mixtures of wide dynamic range of proteomes. The key feature to the abP is its interdisciplinary protocol, which primarily involves chemical, biological and bioanalytical approaches. Interestingly, abP has shaped itself as a standard strategy to sort out the issues related to the activity of enzymes in complex proteomes in vitro as well as in vivo since it visualizes (e.g., using fluorescent dye) the active forms of particular enzymes selectively using small-molecule probes, called as activity-based probes (abPs).
Thus, abPs target the active site of specific protein in a mechanism-based manner and eventually label only the catalytically active form of the enzyme, usually in a covalent fashion. Broadly, abPs subdivided into three essential structural elements: a reactive subgroup, which reacts with the active site of the targeted enzyme and chemically, it is often electrophilic in nature and attacked by the nucleophilic active site; a recognition subgroup, which imposes selectivity upon the reactive subgroup for a specific enzyme class(es) and, chemically it is usually a small chain hydrophilic polyethylene glycol moiety (as a spacer) for keeping a distance between the reactive subgroup and the detectable subgroup; and a detectable subgroup that allows the enrichment, identification and visualization of the targeted proteins (enzymes) in the proteomics problem [3]. The reactive subgroup should be sufficiently reactive to intended active site residues and should be unreactive enough not to react with off-target amino acids or any other functionality present in the sample. The hydrophobicity of the spacer can be tailored by derivatization according to the characteristics of the target enzymes and various substrate-binding preferences.

Furthermore, as detectable subgroup, biotin and fluorescent molecules have been used in place of originally used radioactive markers. As advanced versions, the

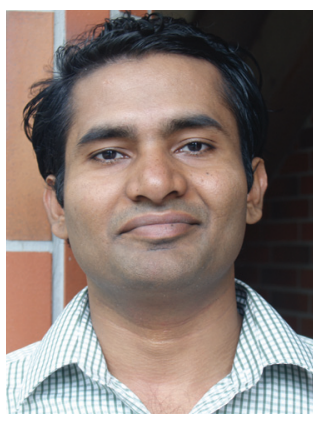

Rituraj Dubey

Department of Chemistry, Buddha Postgraduate College, Kushinagar-274403, India and

Department of Chemistry, Indian Institute of Technology Roorkee, Roorkee-247667, India

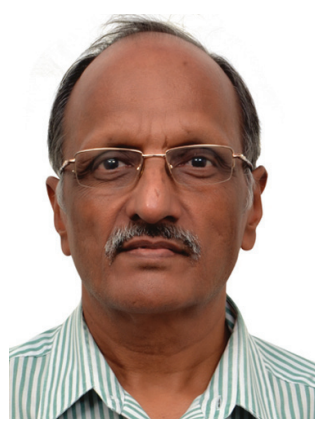

Ravi Bhushan

Author for correspondence: Department of Chemistry, Indian Institute of Technology Roorkee, Roorkee-247667, India and

Institut für Chemie, Carl von Ossietzky Universität Oldenburg, Carl-vonOssietzky-Straße 9-11, D-26129 Oldenburg, Germany rbushfcy54@gmail.com

FUTURE SCIENCE partof 
profiling of enzyme activities has been performed by azide- or alkyne-enabled groups through downstream conjugation using bio-orthogonal reactions such as 'click chemistry' [4] or 'Staudinger-Bertozzi ligation' [5]. Bio-orthogonal reactions are two-step labeling approaches in which modification of abP with a small bio-orthogonal chemical group is followed by modification with a reporter entity. These reactions are used when there is interference in the interaction of abP and enzyme due to the covalent attachment of a fluorescent group or an affinity tag to an abP. However, in the presence of bulky reporter group abP may become less cell-permeable. Generally, 'click chemistry' is a copper(I)-catalyzed $[2,3]$ cycloaddition reaction between an azide and an alkyne [6] and 'Staudinger-Bertozzi ligation' is a chemical reaction between an azide and a phosphine reagent [7]. Thus, we can say that abP has showcased itself as an approach which is long way ahead of conventional proteomics due to proven versatility of abPs as proteomic tools and due to its capability of profiling any fraction of cells/tissues which have even the low-abundant targeted proteins. Moreover, the challenge of keeping an appropriate balance between reactivity and selectivity (i.e., its target-to-background reactivity) of an abP cannot be ignored in order to achieve specific labeling of a desired protein or set of proteins.

\section{"Activity-based proteomics has a promising future in bioanalysis in the background of its inception and the current matured status."}

The next common feature of abP is the visualization, isolation, identification and/or quantification of the labeled proteins. For this purpose, in-gel fluorescence scanning [8] is considered as the most matured bioanalytical platform due to its simplicity, robustness, throughput, sample requirements, rapid quantification of targets of fluorescent abPs and visualization of very low abundant proteins. But, it has a serious limitation as molecular identity to probetagged proteins remained unclear. To overcome this, LC-MS strategy was incorporated as another bioanalytical platform for the identification of protein targets of chemical probes in single step via digestion of probe-treated proteomes with trypsin followed by incubation with affinity based several different type of matrices for the isolation of probe-tagged peptides and then their consecutive elution and analysis by LC-MS/MS [9].

For in-depth proteomics, aiming target detection and identification, the chemical proteomic method was combined with the multidimensional protein identification technology [10] for providing another bioanalytical platform for abP. Capillary electrophoresis has been incepted as bioanalytical platform for abP in order to overcome the relatively high sampleload and low-throughput requirements of LC-MS methods [11]. Moreover, microarray based bioanalytical platforms have proven very valuable tool for abP, since, it has exhibited relatively better resolution and sensitivity in comparison to gel-based bioanalytical platforms since it may provide parallel bioanalysis in proteomics.

This kind of bioanalytical platform has assigned another advantage by revealing molecular identity of probe-tagged proteins (similar to LC-MS-based bioanalytical platforms) via minimizing the throughput and other sample preparation requirements (similar to CEbased bioanalytical platforms). Even then, microarraybased bioanalytical platforms are restricted to special cases of abP due to limited availability of high-quality antibodies and due to limited prior knowledge of the protein targets of probes.

The abP has a promising future in bioanalysis in the background of its inception and the current matured status. Armed with the knowledge of synthetic, analytical and medicinal chemistry, and the knowledge of biology at molecular and cellular levels including proteins, abP is uniquely suitable to meet the challenges of bioanalytical scientists by providing powerful tools and assays for universal analysis of protein expression and function since most proteins do not possess readily available high-affinity-based entities.

\section{Future perspective}

The field of abP has emerged, matured and developed as an indispensable bioanalysis technique for sensitive, selective and rapid profiling of a wide variety of enzymatic activities in complex proteomes in last couple of decades. Existence of wide range of chemical probes, in the present time, has opened the hands of scientists to take its advantage in the functional characterization of many diseases, for example, oncology, metabolic disorders and several other infectious diseases. Our future speculation lies in expansion of abP in the untouched fields of drug discovery, in vivo imaging and studies of structural proteins and protein receptors. We also see, in the near future, the speedy and accurate solutions to hidden secrets of cell function and regulation will not be a challenge to bioanalysis. The recent advancements in the technology of LC-MS will definitely take abP up to a new height in the field of bioanalysis.

\section{Acknowledgements}

The authors would like to thank A von Humboldt-Stiftung for awarding a fellowship for 'renewed research stay in Germany'. 


\section{Financial \& competing interests disclosure}

The authors have no relevant affiliations or financial involvement with any organization or entity with a financial interest in or financial conflict with the subject matter or materials discussed in the manuscript. This includes employ- ment, consultancies, honoraria, stock ownership or options, expert testimony, grants or patents received or pending, or royalties

No writing assistance was utilized in the production of this manuscript.

\section{References}

1 Evans MJ, Cravatt BF. Mechanism-based profiling of enzyme families. Chem. Rev. 106(8), 3279-3301 (2006).

2 Fonovic M, Bogyo M. Activity based probes for proteases: application to biomarker discovery, molecular imaging and drug screening. Curr. Pharm. Des. 13(3), 253-261 (2007).

3 Willems LI, Overkleeft HS, van Kasteren SI. Current developments in activity-based protein profiling. Bioconjug. Chem. 25(7), 1181-1191 (2014).

4 Kolb HC, Finn MG, Sharpless KB. Click chemistry: diverse chemical function from a few good reactions. Angew. Chem. Int. Ed. Engl. 40(11), 2004-2021 (2001).

5 Ovaa H, van Swieten PF, Kessler BM et al. Chemistry in living cells: detection of active proteasomes by a two-step labelling strategy. Angew. Chem. Int. Ed. Engl. 42(31), 3626-3629 (2003).

6 Rostovtsev VV, Green LG, Fokin VV, Sharpless KB. A stepwise huisgen cycloaddition process: copper(I)-

catalyzedregioselective "ligation" of azides and terminal alkynes. Angew. Chem. Int. Ed. Engl. 41(14), 2596-2599 (2002).

7 Saxon E, Bertozzi CR. Cell surface engineering by a modified Staudinger reaction. Science 287(5460), 2007-2010 (2000).

8 Patricelli MP, Giang DK, Stamp LM, Burbaum JJ. Direct visualization of serine hydrolase activities in complex proteomes using fluorescent active site-directed probes. Proteomics 1(9), 1067-1071 (2001).

9 Adam GC, Burbaum JJ, Kozarich JW, Patricelli MP, Cravatt BF. Mapping enzyme active sites in complex proteomes. J. Am. Chem. Soc. 126(5), 1363-1368 (2004).

10 Washburn MP, Wolters D, Yates JR 3rd. Large-scale analysis of yeast proteome by multidimensional protein identification technology. Nat. Biotechnol. 19(3), 242-247 (2001).

11 Okerberg ES, Wu J, Zhang B et al. High-resolution functional proteomics by active-site peptide profiling. Proc. Natl Acad. Sci. USA 102(14), 4996-5001 (2005). 